\title{
New evidence defining the pathology and pathogenesis of lower esophageal sphincter damage
}

\author{
Parakrama Chandrasoma
}

Received: 30 September 2019 / Accepted: 2 October 2019 / Published online: 6 November 2019 (C) The Author(s) 2019

\begin{abstract}
Summary
Background Present diagnosis and management of gastroesophageal reflux disease (GERD) has resulted in a dramatic increase in the incidence of esophageal adenocarcinoma. This is due to failure to identify pathologic changes of early GERD; at present, pathology is limited to management of Barrett esophagus (BE).

Methods Convincing evidence have confirmed that cardiac mucosa distal to the squamocolumnar junction in the endoscopically normal person is a metaplastic GERD-induced esophageal epithelium, and not a normal proximal gastric epithelium.

Results When cardiac mucosa is recognized as a metaplastic esophageal epithelium, it becomes selfevident that the present endoscopic definition of the gastro-esophageal junction is incorrect, and there exists a dilated distal esophagus (DDE) in what is incorrectly termed the "gastric cardia" presently mistaken for proximal stomach. It also becomes clear that the length of the DDE correlates with the presence and severity of GERD and represents the pathology of the entire spectrum of GERD. Further, it allows recognition that the DDE, measured as the gap between esophageal squamous epithelium and gastric oxyntic mucosa that is composed of cardiac mucosa, represents the pathologic anatomy of damage to the abdominal segment of the lower esophageal sphincter (LES).
\end{abstract}

\footnotetext{
P. Chandrasoma, M.D. ( $\square)$

Keck School of Medicine, University of Southern California,

Los Angeles, USA

Los Angeles County, Los Angeles, USA

University of Southern California Medical Center, Los

Angeles, USA

ptchandr@usc.edu
}

Conclusion The new understanding of the significance of cardiac mucosa provides a new and highly accurate histologic method of assessment of LES damage, the primary cause of GERD. This opens a new door to complete histologic assessment of GERD from its etiologic standpoint and to new research that permit early diagnosis of GERD at its outset. Ultimately, such early diagnosis has the potential to reverse the increasing trend of esophageal adenocarcinoma.

Keywords Gastroesophageal reflux disease . Histopathologic diagnosis - Cardiac mucosa $\cdot$ Dilated distal esophagus $\cdot$ Barrett esophagus

\section{Introduction}

Gastroesophageal reflux disease (GERD) is a common human disease. In the Western world, it has a prevalence of $30 \%$ [1]. The cause of GERD is conceptually simple: it results when the mechanism that normally prevents reflux of gastric contents into the esophagus fails. That mechanism is the lower esophageal sphincter (LES). In a patient with a competent LES, reflux does not occur.

Despite this obvious etiology, present medical management is largely aimed at suppressing gastric acid secretion with a naïve and incorrect belief that the disease will be cured by making the refluxed gastric juice less acidic. In fact, the common name for GERD is "acid reflux disease." No amount of acid in the stomach will cause GERD if the LES is competent. No amount of acid suppression will cure reflux if the LES is defective. Patients with GERD who are treated with acid suppressive therapy continue to reflux gastric contents of higher $\mathrm{pH}$, i.e., weak acid reflux [2].

Acid-suppressive drugs, while healing erosive esophagitis and controlling heartburn [3], have not prevented progression of GERD to Barrett esopha- 
gus (BE). Esophageal adenocarcinoma, which always results as a complication of GERD, increased sevenfold in the Western world from 1973 to 2010 and it continues to increase [4].

If acid suppression can claim to heal erosive esophagitis and control heartburn, we must explain how and why it has failed to protect the progression of GERD to adenocarcinoma. The Pro-GERD study in Europe provides insight into the progression of GERD [5, 6]. Over a 5-year period of endoscopic surveillance, there was excellent control of erosive esophagitis. However, 9.7\% of patients without BE at the index endoscopy had developed BE at 5 years. The incidence was significantly associated with the severity of erosive esophagitis at the index endoscopy and regular PPI (proton pump inhibitors) use compared to intermittent PPI use or use of $\mathrm{H}_{2}$ receptor antagonists [6]. It has been suggested that the association with regular PPI use was the result of indication bias. However, indication bias is unlikely to be the full explanation because no significant association was present between severity of symptoms in detailed questionnaires and the occurrence of $\mathrm{BE}$.

Whether or not PPI use is responsible, the fact that $10 \%$ of GERD patients under standard GERD therapy in the primary care setting develop BE in 5 years cannot be disputed. In a disease with a prevalence of $30 \%$ in the population, the conversion to its premalignant complication can explain the rapid increase in the incidence of esophageal adenocarcinoma over the past 30 years. With no changes in the way GERD is managed, the probability is that the increasing incidence of esophageal adenocarcinoma will continue.

\section{Methods}

This review updates recent evidence regarding the pathogenesis, pathology, and pathophysiology of GERD, BE, and esophageal adenocarcinoma, using PUBMED, Google scholar, Scropus, and Springer Link. Statistics were not applied.

\section{Review}

\section{Present understanding of normal anatomy and histology}

The present management of GERD is based on the present understanding of the normal anatomy and histology of the esophagus, LES, and proximal stomach (Fig. 1). At endoscopy, the esophagus is a tube that is lined entirely by squamous epithelium. At the end of the tube, the esophagus flares into what is believed to be the saccular stomach. The stomach is lined by columnar epithelium with longitudinal rugal folds. Normally, the rugal folds reach the horizontal line of the squamocolumnar junction (SCJ) at the point of flaring of the esophagus. The normal SCJ is below the diaphragm because the distal $2-4 \mathrm{~cm}$ of the

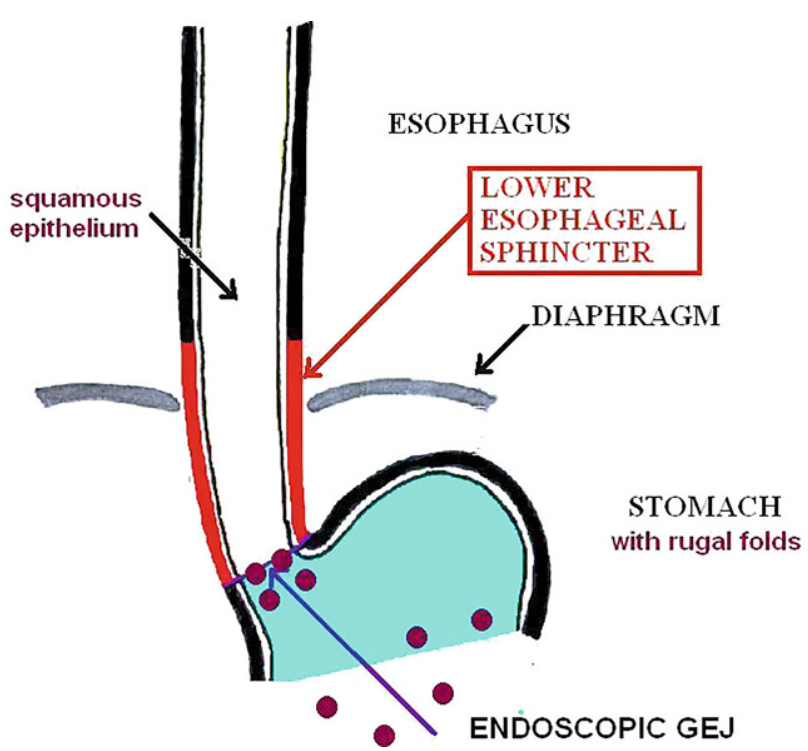

Fig. 1 Diagram of esophagus showing the presently accepted normal state. The entire esophagus is tubular and lined by squamous epithelium (white). The stomach is lined by columnar epithelium with rugal folds (b/ue), histologically of cardiac (proximal $30 \mathrm{~mm}$ ), oxyntic (body), and antral type. The lower esophageal sphincter surrounds the distal $50 \mathrm{~mm}$ of the esophagus, including the entire abdominal esophagus. The circles show the biopsies taken in the DeMeester biopsy protocol in the endoscopically normal person. These include biopsies at the squamocolumnar junction (SCJ), biopsies within $10 \mathrm{~mm}$ distal to the SCJ, and biopsies in the distal body and antrum

esophagus are in the abdomen. When a sliding hiatal hernia is present, the SCJ is above the diaphragm; in these patients, the gastroesophageal junction is defined as the proximal limit of rugal folds. When a patient has BE, the SCJ is displaced cephalad and the GEJ is defined as the point of flaring of the tubular esophagus, which is concordant with the proximal limit of rugal folds (Fig. 2).

The distal $5 \mathrm{~cm}$ of the esophagus is surrounded by the lower esophageal sphincter (Fig. 1), a high-pressure zone designed to prevent reflux along the pressure gradient that exists from the stomach (intraluminal pressure $+5 \mathrm{mmHg}$ ) to the mid-thoracic esophagus (-5 mmHg).

The normal histology, as it is presently believed, is that the entire esophagus is lined by squamous epithelium and transitions to the stomach at the proximal limit of rugal folds. It is believed that the proximal stomach has a variable amount of cardiac mucosa distal to the endoscopic GEJ followed by gastric oxyntic epithelium. Hayward, in 1961 [7], stated without evidence that the proximal $30 \mathrm{~mm}$ of the stomach was normally lined by cardiac mucosa. He suggested that this mucus-secreting epithelium acted as a buffer zone that prevents squamous epithelial digestion by gastric acid.

The present guidelines for endoscopy and biopsy are based on this understanding of anatomy and his- 


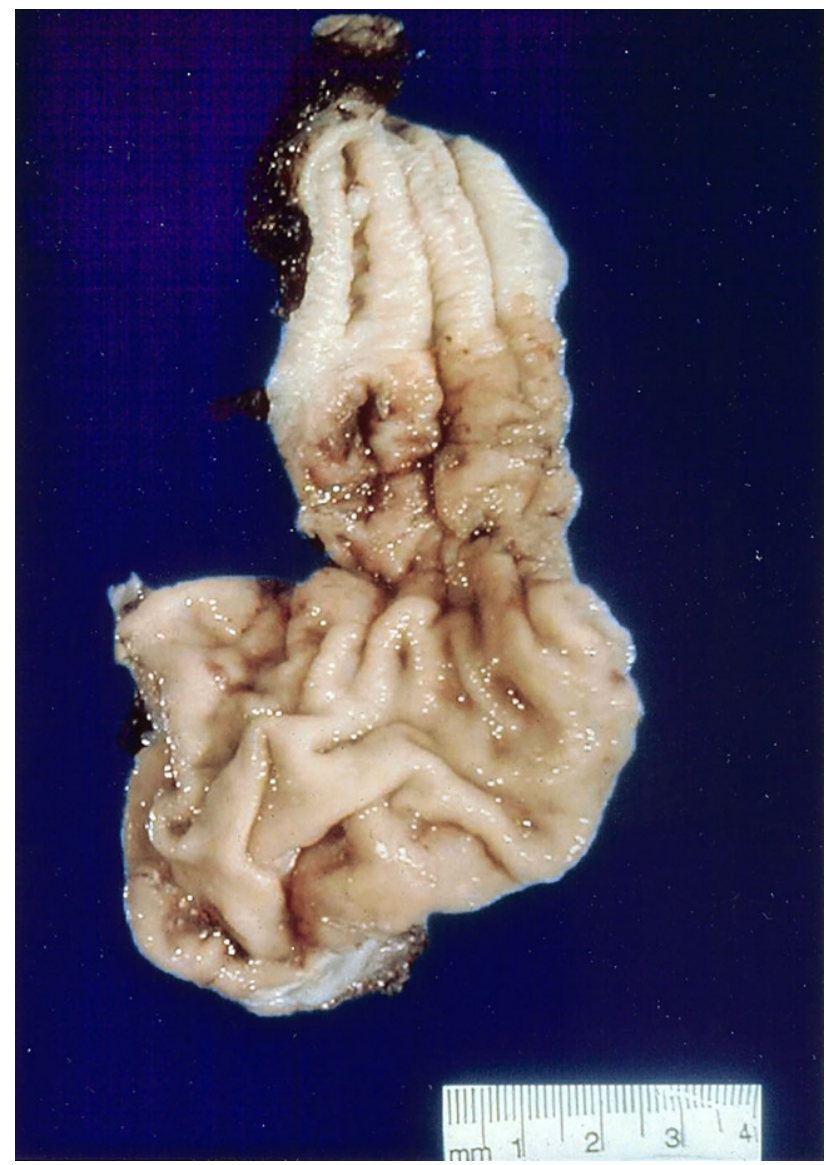

Fig. 2 The present interpretation of the gross pathology and endoscopy in a patient with long-segment Barrett esophagus with adenocarcinoma. The esophagus is a tube that flares into the saccular stomach at a point that is concordant with the proximal limit of rugal folds, which defines the gastroesophageal junction. The esophagus is lined proximally by squamous epithelium and distally by Barrett-type columnar epithelium. In such a patient, the DeMeester protocol will take biopsies at $1-2 \mathrm{~cm}$ intervals from the Barrett segment in addition to the biopsies taken in a person with normal endoscopy

tology. If the patient with GERD has no abnormality at endoscopy, biopsies are not recommended because the epithelium distal to the normal SCJ where it meets the proximal limit of rugal folds is gastric $[3,8]$. Biopsy is only indicated if there is a visible columnar-lined esophagus (CLE) [3, 8]; the objective of biopsy is to identify intestinal metaplasia, which defines $\mathrm{BE}$ and is an indication for endoscopic surveillance because it is premalignant. The GERD patient who does not have BE has not been studied histologically because of the failure to take biopsies before BE has developed.

\section{The basic flaw in the present understanding}

In 1990, Dr. Tom DeMeester established a clinical program in foregut surgery at the University of Southern California. A unique element of this program was an endoscopic biopsy protocol that he used for all patients undergoing upper gastrointestinal endoscopy.
In persons with normal endoscopy, sets of biopsies were taken from the SCJ, from within $10 \mathrm{~mm}$ distal to the SCJ, and from the distal gastric body and antrum (Fig. 1).

Over the next three decades, over 15000 patients undergoing endoscopy at the unit had histologic assessment based on specimens from this biopsy protocol. At the weekly clinicopathologic conferences of the unit, it quickly became apparent that the traditional belief that cardiac mucosa normally lined $30 \mathrm{~mm}$ of the proximal stomach was incorrect. This was initially the result of two observations: (a) cardiac mucosa was sometimes absent in the two biopsies at and within $10 \mathrm{~mm}$ distal to the endoscopic GEJ, and (b) when present, the amount of cardiac mucosa and inflammation within it correlated directly with the severity of GERD.

These observations led to a clinical study that correlated cardiac mucosa to objective criteria for GERD. This resulted in an abstract in 1994 [9] followed by a larger study in 1997. In that study of 334 patients, Oberg et al. [10] showed that patients who had cardiac mucosa in the biopsies distal to a normal SCJ had a significantly higher probability of an abnormal 24hour $\mathrm{pH}$ test and abnormalities in the LES compared to those who did not.

The medical establishment resistance to the finding that cardiac mucosa was a GERD-related pathologic finding contrary to the traditional and universal view that it was normal proximal gastric mucosa was intense. The finding was ignored by most, but stimulated numerous important studies over the next two decades. Mainstream belief about cardiac mucosa has evolved towards an acceptance of the concept. At present, most pathologists still incorrectly believe that a small amount (less than $4 \mathrm{~mm}$ ) of cardiac mucosa normally lines the proximal stomach. However, there is evidence that the new concept is very close to mainstream acceptance. In a review of Barrett esophagus in the August 2019 issue of Gastroenterology, Stuart Spechler's group writes [11]: “... there is considerable indirect evidence to support a hypothesis, proposed by Chandrasoma in 1997, that cardiac mucosa is not normal but an acquired, GERD-induced metaplasia, and that cardiac mucosa is the precursor of intestinal metaplasia in BE."

As soon as the fatal flaw that cardiac mucosa is normal in the proximal stomach is corrected, the definition of the GEJ will change and the early changes of GERD in the most distal abdominal esophagus will be recognized. These changes represent the pathologic changes associated with abdominal LES damage, the primary cause of GERD. At present, because of the incorrect definition of the GEJ at endoscopy as the proximal limit of rugal folds, the critical early changes of GERD are mistaken for normal proximal stomach. 


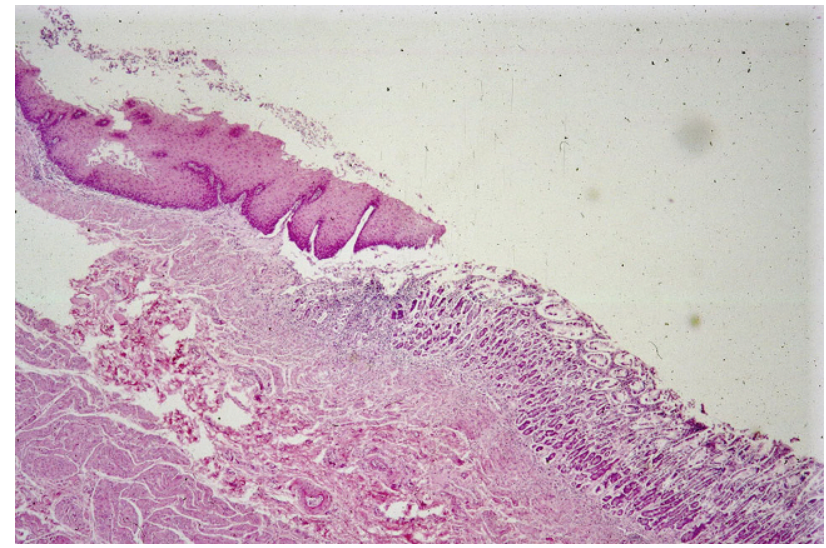

Fig. 3 The zero squamo-oxyntic gap in a child with no gastroesophageal reflux disease at autopsy. The esophageal squamous epithelium transitions at the squamocolumnar junction to gastric oxyntic mucosa with straight tubular glands composed of parietal and chief cells. There is no cardiac mucosa

\section{Evidence that cardiac mucosa is a metaplastic esophageal and not a normal proximal gastric mucosa}

\section{Cardiac mucosa is not present at the SCJ in all} persons

In a study of autopsies in 18 persons without clinical GERD during life [12], we showed that pure cardiac mucosa was absent in 10/18 (56\%). Oxyntocardiac mucosa (=cardiac mucosa with parietal cells in the glands) was present in all persons. However, both cardiac and oxyntocardiac mucosae were absent in some part of the circumference of the SCJ in $9 / 18$ (50\%). This proves that cardiac mucosa is absent in some persons in some part of the circumference of the GEJ. In these persons, the esophageal squamous epithelium transitions directly to gastric oxyntic mucosa (the zero squamo-oxyntic gap [13]; Fig. 3).

Studies from other centers have disputed the finding that cardiac mucosa can be absent. Kilgore et al. [14], in an autopsy study, showed that cardiac mucosa was present in all persons to a length of 1-4 $\mathrm{mm}$. Marsman et al. [15], in an endoscopic study, reported that cardiac mucosa was present at the normal squamocolumnar junction in $62 \%$ of patients; in the other $38 \%$, the squamous epithelium transitioned to oxyntocardiac mucosa. No patient in these studies had a zero squamo-oxyntic gap. It should be understood that the absence of cardiac mucosa is not proved by these findings. A single photograph (Fig. 3) of a zero squamo-oxyntic gap overrides claims that cardiac mucosa is universal.

\section{When present, cardiac mucosa correlates with presence of GERD}

The study of Oberg et al. [10] established the relationship of the presence of cardiac mucosa with abnormal reflux and LES abnormalities. Cardiac mucosa

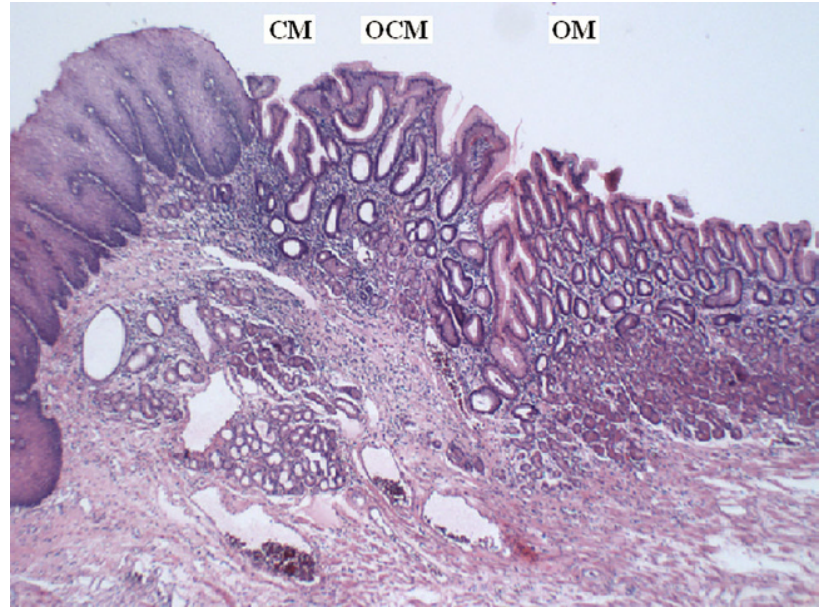

Fig. 4 A 2 mm squamo-oxyntic gap in a resected specimen of a patient with squamous carcinoma of the esophagus. The squamous epithelium has been displaced cephalad (to the left in the picture) by cardiac and oxyntocardiac epithelium that separates it from the proximal limit of gastric oxyntic mucosa. Note the disorganized mucous cell containing glands in cardiac mucosa and the presence of inflammation and reactive changes in the foveolar region ("carditis"). CM cardiac mucosa, OCM oxyntocardiac mucosa, OM oxyntic mucosa

between the SCJ and gastric oxyntic mucosa (i.e., in the squamo-oxyntic gap) is therefore a GERD-induced metaplasia of squamous epithelium of the distal abdominal esophagus (Fig. 4). When found, cardiac mucosa always shows chronic inflammation (Fig. 4). Carditis is the earliest specific histologic manifestation of GERD [16].

The length of cardiac mucosa distal to the endoscopic GEJ correlates with severity of GERD

Glickman et al. [17] showed that the presence of $>1 \mathrm{~mm}$ of cardiac mucosa in children was associated with a significantly higher prevalence of reflux esophagitis in the squamous epithelium compared with patients with $<1 \mathrm{~mm}$. Chandrasoma et al. [18] showed a significantly higher acid exposure in patients with $>20 \mathrm{~mm}$ of cardiac mucosa compared to patients with $<20 \mathrm{~mm}$. Chandrasoma et al. [19] showed, in esophagectomy specimens, that patients with squamous carcinoma had lengths of cardiac mucosa similar to the non-GERD autopsy population $(<5 \mathrm{~mm})$, whereas patients with GERD-induced adenocarcinoma had lengths of $10-20 \mathrm{~mm}$.

The pathogenesis of cardiac metaplasia has been elucidated

In two elegant studies of asymptomatic volunteers, Ayazi et al. [20] from our unit and Robertson et al. [21], from Professor McColl's unit in Glasgow, showed that gastric over-distension caused by air insufflation [20] or a heavy meal [21] resulted in a temporary shortening of the LES that caused the squamous epithelium of the distal esophagus to become exposed to gastric contents. With cumulative exposure, Derakhshan 
et al. [22] showed that the squamous epithelium underwent increasing expression of trefoil family factor 3 (TFF3) that triggered metaplasia into cardiac mucosa [22]. This study also showed that the small amounts of cardiac mucosa found in these asymptomatic volunteers was similar to non-intestinalized columnar epithelium in BE [22].

Robertson et al. [21] also showed that the length of cardiac mucosa was significantly shorter (median of $1.75 \mathrm{~mm}$ ) in volunteers without central obesity than in obese persons (median $2.5 \mathrm{~mm}$ ). The correlation between cardiac mucosal length and central obesity suggest that over-eating is a common etiology for these two entities. Bu et al. [23] showed that there was a dose-related relationship between the presence of cardiac mucosa and body mass index.

These conclusively show the mechanism whereby the most distal squamous epithelium of the abdominal esophagus in normal persons undergoes metaplasia into cardiac mucosa due to exposure to acid during gastric overdistention. The presence of a pathogenesis for the millimetric amounts of cardiac mucosa present at the SCJ conclusively proves that it is not a normal proximal gastric mucosa.

\section{The definition of the gastroesophageal junction}

When it is accepted that cardiac mucosa distal to the endoscopic GEJ (defined as the proximal limit of rugal folds) is a metaplastic esophageal epithelium derived from GERD-induced damage to the squamous epithelium, it must follow that the proximal limit of rugal folds is not the true GEJ. The evidence that the proximal limit of rugal folds is the true GEJ is a single unconvincing and flawed report by McClave et al. in 1987 [24]. It must be rejected in the light of presence evidence. The true GEJ is the junction between metaplastic cardiac and oxyntocardiac mucosa with gastric oxyntic epithelium [19]. It can be defined only by histology.

The measured length of cardiac mucosa distal to the endoscopic GEJ in a limited number of resected and autopsy specimens is zero to $28 \mathrm{~mm}[12,25]$. When more studies are done, the length is likely to be greater. This length of cardiac mucosa is mistaken at endoscopy for proximal stomach because of the erroneous use of the proximal limit of rugal folds to define the GEJ at endoscopy.

In a study of resected specimens, Chandrasoma et al. [19] showed that the length of cardiac and oxyntocardiac mucosa distal to the proximal limit of rugal folds was $<5 \mathrm{~mm}$ in two patients with squamous carcinoma and between 10 and $20.5 \mathrm{~mm}$ in 8 patients with GERD-induced adenocarcinoma. Mapping of esophageal submucosal glands showed that the distal extent of the submucosal glands was concordant with the distal limit of cardiac and oxyntocardiac mucosa (Fig. 5). Submucosal glands were never seen under gastric oxyntic mucosa. This finding proves that car- diac and oxyntocardiac mucosa distal to the proximal limit of rugal folds is esophageal.

In that study, the anatomy of the region distal to the endoscopic GEJ that was lined by cardiac and oxyntocardiac mucosa was dilated and its mucosa was lined by rugal folds. This was termed the dilated distal esophagus [26] and defined as that area distal to the end of the tubal esophagus that is dilated, lined by cardiac and oxyntocardiac mucosa, and contains rugal folds (Fig. 5). This exactly mimics the stomach at gross examination and endoscopy; the error is only revealed by the histologic presence of metaplastic cardiac mucosa.

Korn et al. [27] measured the circumference at the anatomic GEJ, which is marked accurately by the peri-

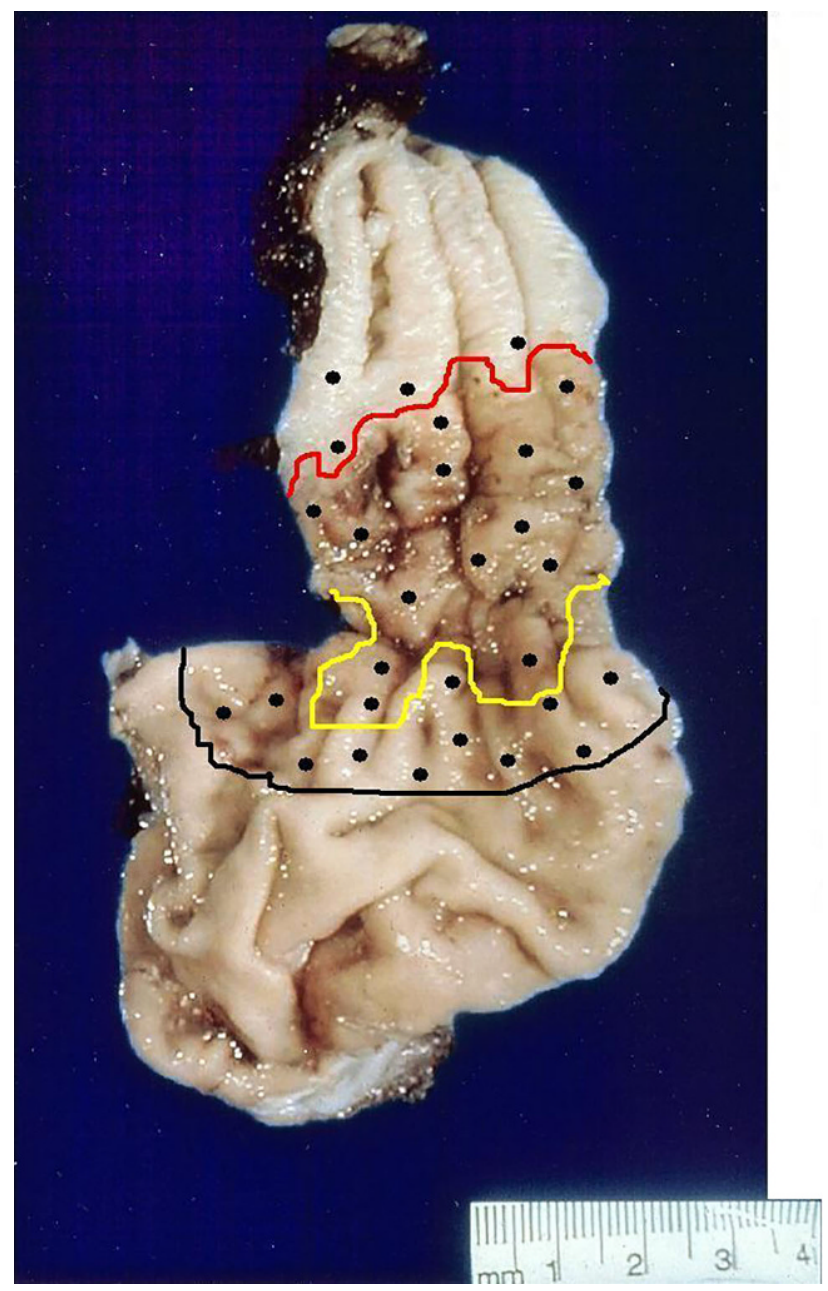

Fig. 5 Patient in Fig. 2 showing the distribution of metaplastic columnar epithelium in the esophagus. The red line is the squamocolumnar junction; the yellow line is the distal extent of intestinal metaplasia. Cardiac and oxyntocardiac epithelia extend $20 \mathrm{~mm}$ distal to the proximal limit of rugal folds (black line). The black dots represent mapping of the submucosal glands in the specimen; their distribution is concordant with the extent of cardiac and oxyntocardiac epithelium, proving that the area distal to the proximal limit of rugal folds is esophageal ("the dilated distal esophagus"). The true GEJ (Gastro-esophageal junction) is defined histologically as the proximal limit of rugal folds 
Fig. 6 Diagram showing why the abdominal esophagus dilates when it loses the protection of the high-pressure zone by lower esophageal sphincter damage. The sphincterless abdominal esophagus can no longer resist the dilatory tendency of the positive intraluminal pressure which is accentuated with every meal. LES lower esophageal sphincter, CLE columnar lined esophagus

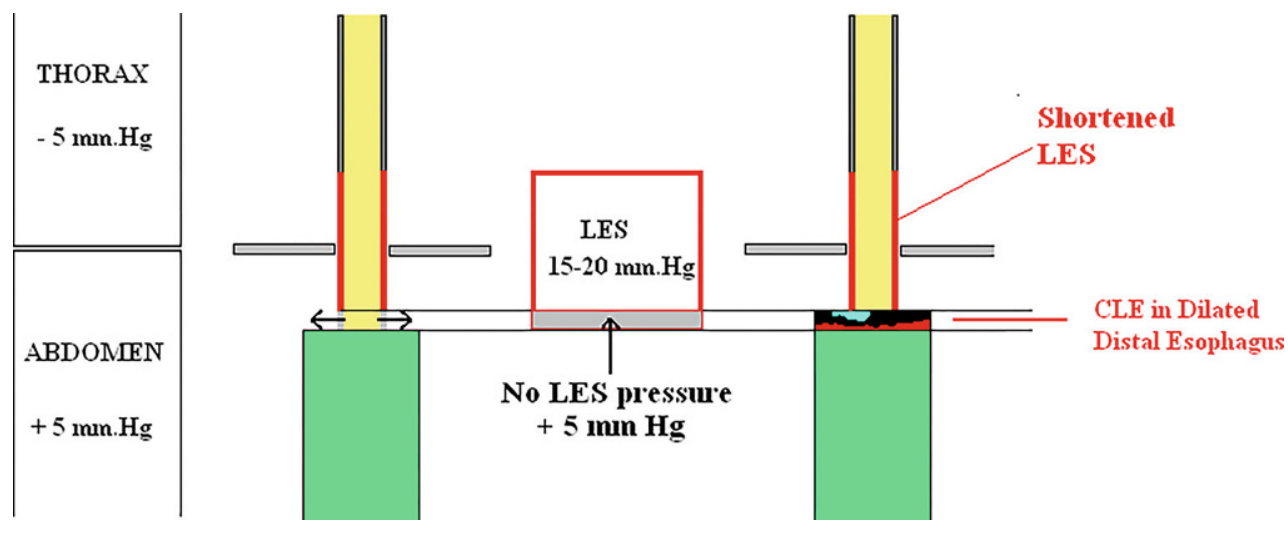

toneal reflection, at surgery. They showed that the mean circumference in persons without GERD was 6.3 , that of GERD patients without $B E$ was $8.9 \mathrm{~cm}$, and that of patients with $\mathrm{BE}$ was $13.8 \mathrm{~cm}$. This confirms the increasing dilatation of the distal esophagus with increasing GERD severity.

What causes dilatation of the esophagus when cardiac metaplasia of squamous epithelium occurs?

The entire abdominal esophagus is normally surrounded by the high-pressure zone of the abdominal segment of the LES. This high pressure is necessary to withstand the dilatory force of the positive intraluminal pressure in the abdominal esophagus and maintain its tubular shape (Fig. 6).

Normal LES tone is likely maintained by a local reflex arc with the afferent nerve endings that are plentiful in the squamous epithelium [28], a central relay in the ganglion cells of the submucosal and myenteric plexus, and the efferent nerves passing to the smooth muscle to maintain tonic contraction. When cardiac metaplasia occurs in the esophagus, the nerve endings in the squamous epithelium are lost, the afferent arc of the local reflex is disrupted, and the high-pressure of LES tone disappears. With loss of LES tone, the abdominal esophagus that has undergone cardiac metaplasia dilates because of the positive intraluminal pressure and the fact that it distends with every gastric over-distension (Fig. 6). It essentially becomes "gastricized," behaving physiologically like the stomach, distending and collapsing in the full and fasting state, respectively. This results in the development of rugal folds which are a feature of all reservoir organs.

\section{Conclusion}

\section{Result of this new understanding}

The abdominal esophagus surrounded by the abdominal segment of the LES is the critical barrier that prevents reflux of gastric juice from the esophagus. The only recognized measurement of the LES that is presently used for diagnosis is the manometric length of the functioning LES. It is known that LES shortening occurs in GERD. However, without knowledge of its original length, manometric length of the abdominal LES cannot define LES shortening. Lee et al. [29] showed that in patients with clinical GERD, the length of the abdominal segment of the LES bears an inverse linear relationship to acid exposure by the 24-hour $\mathrm{pH}$ test. In that study, the length of the abdominal segment of the LES decreased from 18 to $3 \mathrm{~mm}$ with increasing reflux [29]. Zaninotto et al. [30] showed that an abdominal LES length of $<10 \mathrm{~mm}$ is indicative of a defective LES and is associated with an abnormal 24-hour pH test. This criterion for an abnormal LES is of value to identify an LES that needs to be surgically repaired. There is no criterion of manometric LES length or pressure that identifies early GERD prior to a defective LES. Surely, the change of a normal LES to a defective LES is not an acute and sudden event. It

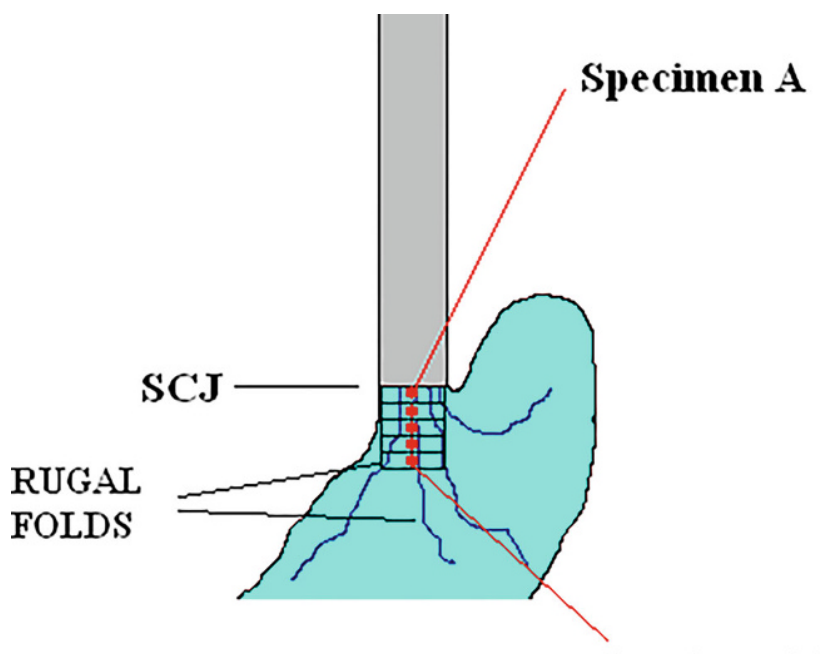

Specimen E

Fig. 7 Multi-level biopsy protocol for assessment of the length of cardiac and oxyntocardiac mucosa distal to the endoscopic gastroesophageal junction (= squamocolumnar junction [SCJ] in the endoscopically normal person). Five sets of biopsies are at $5 \mathrm{~mm}$ intervals at and distal to the SCJ and submitted in separate jars (red dots). Though unoriented, these biopsies provide reasonable assessment of the length of cardiac mucosa 
is highly likely that there is a chronic progressive loss of LES length before the defective state is reached. The chronic progression is likely to be chronic repetitive gastric over-distension due to chronic over-eating over a long period. Kahrilas et al. [31] showed that the temporary LES shortening associated with gastric over-distension by air insufflation was identical irrespective of baseline length of the LES. This points to the probability of a linear progression of LES shortening.

The relationship between metaplastic cardiac mucosa distal to the endoscopic GEJ and concordant dilatation of the esophagus in the area of the damaged LES enables a simple formula to be developed regarding the abdominal esophagus:

\section{Length of abdominal esophagus}

(= length of abdominal segment of the LES)

$$
\begin{aligned}
= & \text { Length of the tubular abdominal esophagus } \\
& =\text { manometric length of the functional } \\
& \text { abdominal segment of the LES) }
\end{aligned}
$$

+ Length of the dilated distal esophagus/cardiac mucosa distal to endoscopic GEJ

(= length of damaged/shortened abdominal segment of the LES)

Measurement of the length of cardiac and oxyntocardiac mucosa in the dilated distal esophagus is an accurate pathologic assessment of abdominal LES damage. This new pathologic test measures the critical causative factor in determining the absence, presence, and severity of GERD. It is analogous to coronary artery narrowing as a more accurate assessment of ischemic heart disease than symptoms of ischemia.

Accurate measurement of the length of cardiac mucosa cannot be made with present biopsy technology. Biopsy forceps in use can access an unoriented pinch sample of the mucosa measuring a maximum of 8 $\mathrm{mm}$ [21]. Multilevel biopsies distal to the endoscopic GEJ (Fig. 7), though they may lack precision of measurement, provide the best possible measurement of the length of the dilated distal esophagus with present biopsy technology [32]. A quantitative biopsy device that can remove a $20-25 \mathrm{~mm}$ longitudinal piece of mucosa vertically on the lesser curvature distal to the endoscopic GEJ (the SCJ in a patient without BE) needs to be developed. This will provide an accurate measurement of abdominal LES damage.

The available data suggest that a length of dilated distal esophagus $<15 \mathrm{~mm}$ represents a competent LES and absence of GERD. This minimal level of abdominal LES damage is almost universal, producing small amounts of cardiac metaplasia. Such minimal damage is within the reserve capacity of the LES and does not result in LES failure or reflux. The changes of GERD are limited to the area of the damaged distal abdominal LES (intrasphincteric GERD).

When LES damage (cardiac mucosal length) reaches

\begin{tabular}{|c|c|c|c|}
\hline & STAGE OF GERD & $\begin{array}{c}\text { REFLUX } \\
\text { 24-hour pH Test }\end{array}$ & $\begin{array}{l}\text { CELLULAR } \\
\text { CHANGES }\end{array}$ \\
\hline $\begin{array}{l}35 \mathrm{~mm} \\
30 \mathrm{~mm}\end{array}$ & Severe GERD & $\begin{array}{l}\mathrm{pH}<4 \\
>4.5 \%\end{array}$ & $\begin{array}{l}\text { Presence or } \\
\text { Risk of Visible CLE } \\
\text { Erosive esophagitis } \\
\text { CE in DDE }\end{array}$ \\
\hline $20 \mathrm{~mm}$ & Mild GERD) & $\begin{array}{c}\mathrm{pH}<4 \\
>1.1 \text { to } 4.5 \%\end{array}$ & $\begin{array}{l}\text { Erosive esophagitis } \\
\text { NERD } \\
\text { CE linited to DIE }\end{array}$ \\
\hline $15 \mathrm{mut}$ & $\begin{array}{l}\text { Phase of compensated LES } \\
\text { damage }\end{array}$ & $\begin{array}{c}\mathrm{pH}<4 \\
\text { zero to } 1.1 \%\end{array}$ & CE linited to $\mathrm{DDE}$ \\
\hline $5 \mathrm{~mm}$ & $\begin{array}{l}\text { Phase of compensated LES' } \\
\text { damage }\end{array}$ & zero & CE limited to $\mathrm{DDF}$ \\
\hline $0 \mathrm{~mm}$ & & & \\
\hline
\end{tabular}
a length $>15 \mathrm{~mm}$, clinical evidence of early postprandial GERD becomes appears. With a further progres-
Fig. 8 Diagram showing the correlation between damage to the lower esophageal sphincter and newly understood stages of gastroesophageal reflux disease. The first stage, where LES damage is within its reserve capacity, is not associated with reflux because the LES is competent. As LES damage progressively shortens the abdominal LES from its dis- tal end, there is progression from postprandial reflux to fully established reflux disease with a defective lower esophageal sphincter. GERD gastroesophageal reflux disease, NERD nonerosive reflux disease, CE cardiac epithelium, CLE columnar lined esophagus, LES lower esophageal sphincter, DDE dilated distal esophagus 
sive increase of LES damage, the severity of GERD progressively increases [29] until the LES becomes defective and associated with an abnormal $\mathrm{pH}$ test at $\mathrm{a}<10 \mathrm{~mm}$ length of the abdominal LES, to the endpoint of destruction of the entire abdominal segment of the LES when reflux is severe, impossible to control with medical therapy, and likely to be associated with BE ([33]; Fig. 8).

The measurement of abdominal LES damage by histology is possible in the asymptomatic person. If abdominal LES damage has a linear progression, this information may allow prediction of GERD and its severity in the person's future, allowing development of new methods to prevent progression of LES damage in selected persons [34].

I (the author), who have no symptoms of GERD, have had multiple biopsies taken at and distal to my normal SCJ at intervals of 5 to $25 \mathrm{~mm}$ distal to the SCJ. Only the biopsy at the SCJ had metaplastic cardiac mucosa; the remainder had normal gastric oxyntic mucosa. My measured dilated distal esophagus was $4 \mathrm{~mm}$ with $<1 \mathrm{~mm}$ of cardiac mucosa and $4 \mathrm{~mm}$ of oxyntocardiac mucosa. If it is correct that abdominal LES shortening has a linear progression, I have calculated that my LES will remain competent until I reach an age of 250 years!

Conflict of interest P. Chandrasoma declares that he has no competing interests.

Open Access This article is distributed under the terms of the Creative Commons Attribution 4.0 International License (http://creativecommons.org/licenses/by/4.0/), which permits unrestricted use, distribution, and reproduction in any medium, provided you give appropriate credit to the original author(s) and the source, provide a link to the Creative Commons license, and indicate if changes were made.

\section{References}

1. El-Serag HB, Sweet S, Winchester CC, et al. Update on epidemiology of gastroesophageal reflux disease: a systematic review. Gut. 2014;63:871-80.

2. Blonski W, Vela MF, Castell DO. Comparison of reflux frequency during prolonged multichannel intraluminal impedance and $\mathrm{pH}$ monitoring on and off acid suppressive therapy. JClin Gastroenterol. 2009;43:816-20.

3. PeterJ, Shaheenb NJ,Vaezic MF.American Gastroenterological Association Institute technical review on the management of gastroesophageal reflux disease. Gastroenterology. 2008;135:1392-413.

4. Pohl H, Sirovich B, Welch HG. Esophageal adenocarcinoma incidence: are we reaching the peak? Cancer Epidemiol Biomarkers Prev. 2010;19:1468-70.

5. Kulig M, Nocon M, Vieth M, et al. Risk factors of gastroesophageal reflux disease: methodology and first epidemiological results of the ProGERD study. J Clin Invest. 2004;57:580-9.

6. Malfertheiner P, Nocon M, ViethM, etal. Evolution of gastrooesophageal reflux disease over 5 years under routine medical care-the ProGERD study. Aliment Pharmacol Ther. 2012;35:154-64.
7. Hayward J. The lower end of the oesophagus. Thorax. 1961;16:36-41.

8. Sharma P, McQuaid K, Dent J, et al. A critical review of the diagnosis and management of Barrett's esophagus: the AGA Chicago Workshop. Gastroenterology. 2004;127:310-30.

9. Clark GWB, Ireland AP, Chandrasoma P, et al. Inflammation and metaplasia in the transitional mucosa of the epithelium of the gastroesophageal junction: a new marker for gastroesophageal reflux disease. Gastroenterology. 1994;106:A63.

10. Oberg S, Peters JH, DeMeester TR, et al. Inflammation and specialized intestinal metaplasia of cardiac mucosa is a manifestation of gastroesophageal reflux disease. Ann Surg. 1997;226:522-32.

11. Que J, Garman KS, Souza RF, Spechler SJ. Pathogenesis and cells of origin of Barrett's esophagus. Gastroenterology. 2019;157:349-64.

12. Chandrasoma PT, Der R, Ma Y, et al. Histology of the gastroesophageal junction: an autopsy study. Am J Surg Pathol. 2000;24:402-9.

13. Chandrasoma PT, Wijetunge S, DeMeester SR, Hagen JA, DeMeester TR. The histologic squamo-oxyntic gap: an accurate and reproducible diagnostic marker of gastroesophageal reflux disease. Am J Surg Pathol. 2010;34:1574-81.

14. Kilgore SP, Ormsby AH, Gramlich TL, et al. The gastric cardia: fact or fiction? Am J Gastroenterol. 2000;95:921-4.

15. Marsman WA, van Sandyck JW, Tytgat GNJ, et al. The presence and mucin histochemistry of cardiactype mucosa at the esophagogastric junction. Am J Gastroenterol. 2004;99:212-7.

16. Der R, Tsao-Wei DD, DeMeester T, et al. Carditis: a manifestation of gastroesophageal reflux disease. Am J Surg Pathol. 2001;25:245-52.

17. Glickman JN, Fox V, Antonioli DA, Wang HH, Odze RD. Morphology of the cardia and significance of carditis in pediatric patients. Am J Surg Pathol. 2002;26:1032-9.

18. Chandrasoma PT, Lokuhetty DM, DeMeester TR, et al. Definition of histopathologic changes in gastroesophageal reflux disease. Am J Surg Pathol. 2000;24:344-51.

19. Chandrasoma P, Makarewicz K, Wickramasinghe K, et al. A proposal for a new validated histologic definition of the gastroesophageal junction. Human Pathology. 2006;37:40-7.

20. Ayazi S, Tamhankar A, DeMeester SR, et al. The impact of gastric distension on the lower esophageal sphincter and its exposure to acid gastric juice. Ann Surg. 2010;252:57-62.

21. Robertson EV, Derakhshan MH, Wirz AA, et al. Central obesity in asymptomatic volunteers is associated with increased intrasphincteric acid reflux and lengthening of the cardiac mucosa. Gastroenterology. 2013;145:730-9.

22. Derakhshan MH, Robertson EV, Lee YY, et al. In healthy volunteers, immunohistochemistry supports squamous to columnar metaplasia as mechanism of expansion of cardia, aggravated by central obesity. Gut. 2015;64:1705-14.

23. Bu X, Ma Y, Der R, DeMeester T, et al. Body mass index is associated with Barrett esophagus and cardiac mucosal metaplasia. Dig Dis Sci. 2006;51:1589-94.

24. McClave SA, Boyce HW Jr, Gottfried MR. Early diagnosis of columnar lined esophagus: a new endoscopic diagnostic criterion. Gastrointest Endosc. 1987;33:413-6.

25. Sarbia M, Donner A, Gabbert HE. Histopathology of the gastroesophageal junction. A study on 36 operation specimens. Am J Surg Pathol. 2002;26:1207-12.

26. Chandrasoma P, Wijetunge S, Ma Y, et al. The dilated distal esophagus: a new entity that is the pathologic basis of early gastroesophageal reflux disease. Am J Surg Pathol. 2011;35:1873-81. 
27. Korn O, Csendes A, Burdiles P, et al. Anatomic dilatation of the cardia and competence of the lower esophageal sphincter: a clinical and experimental study. J Gastrointest Surg. 2000;4:398-406.

28. Rodrigo J, Hernandez CJ, Vidal MA, Pedrosa JA. Vegetative innervation of the esophagus. III. Intraepithelial endings. Acta Anat. 1975;92:242-58.

29. LeeJ, Anggiansah A, Anggiansah R, etal. Effects of age on the gastroesophageal junction, esophageal motility and reflux disease. Clin Gastroenterol Hepatol. 2007;5:1392-8.

30. Zaninotto G, DeMeester TR, Schwizer W, Johansson KE, Cheng SC. The lower esophageal sphincter in health and disease. Am J Surg. 1988;155:104-11.

31. Kahrilas PJ, Shi G, Manka M, Joehl RJ. Increased frequency of transient lower esophageal sphincter relaxation induced by gastric distension in reflux patients with hiatal hernia. Gastroenterology. 2000;118:688-95.
32. Ringhofer C, Lenglinger J, Izay B, et al. Histopathology of the endoscopic esophagogastric junction in patients with gastroesophageal reflux disease. Wien Klin Wochenschr. 2008;120:350-9.

33. Stein HJ, Barlow AP, DeMeester TR, Hinder RA. Complications of gastroesophageal reflux disease. Role of the lower esophageal sphincter, esophageal acid and acid/ alkaline exposure, and duodenogastric reflux. Ann Surg. 1992;216:35-43.

34. Chandrasoma PT. GERD: A New Understanding of Pathology Pathophysiology and Treatment. San Diego: Academic Press; 2018

Publisher's Note Springer Nature remains neutral with regard to jurisdictional claims in published maps and institutional affiliations. 